\title{
5-Aminolevulinic acid (ALA) promotes primary root elongation through modulation of auxin transport in Arabidopsis
}

\author{
Yuyan $\mathrm{An}^{1}$ (D) Danxuan Cheng ${ }^{1} \cdot$ Zhixiong Rao $^{1} \cdot$ Yongping Sun ${ }^{2} \cdot$ Quan Tang $^{2} \cdot$ Liangju Wang $^{1}$
}

Received: 18 March 2018 / Revised: 14 April 2019 / Accepted: 17 April 2019 / Published online: 27 April 2019

(c) The Author(s) 2019

\begin{abstract}
The specific function of 5-aminolevulinic acid (ALA), a new plant growth regulator, in modulating root growth of plants and the mechanisms underlying ALA-regulated root growth are largely unknown. Here, Arabidopsis seedlings were photographed and collected before and after ALA or 2,3,5-triiodobenzoic acid (TIBA) treatment for determination of root growth, fluorescence intensities of PIN1, PIN2, PIN7, and DR5, and gene expression levels of auxin synthesis, signaling, and transport. We first demonstrated that ALA significantly promoted Arabidopsis primary root elongation. We also found that TIBA, an auxin polar transport inhibitor, inhibited ALA-promoted root elongation, indicating that auxin transport is involved in ALAregulated root growth. Then, the observations of PIN1, PIN2, and PIN7 at protein and transcript levels suggest that ALA improves auxin transport mainly through regulating auxin efflux carriers. Furthermore, the expression patterns of auxinresponsive reporter DR5rev:GFP were not correlated well with the expression of $Y U C 2$, a key biosynthetic gene of auxin, but were consistent with changes of PIN1, PIN2, and PIN7. In addition, ALA did not affect the gene expression of auxin receptor, TRANSPORT-INHIBITOR-RESISTANT1 (TIR1). Taken together, we conclude that ALA promotes primary root elongation of young Arabidopsis seedlings mainly through improving auxin transport. Our data suggest the reciprocal interaction between ALA and auxin, providing new insights into the mechanisms underlying ALA-promoted plant root growth.
\end{abstract}

Keywords 5-Aminolevulinic acid $\cdot$ Arabidopsis $\cdot$ Auxin $\cdot$ Polar transport $\cdot$ Root growth

\section{Introduction}

Root, an essential organ of higher plants, not only structurally supports the plant anchoring in the soil, but also uptakes nutrients and water for plants (Slovak et al. 2016). Moreover, roots are also important for its ability of sensing and responding to the changing environments (Tsukagoshi 2016). In addition, roots can also be storage organs for some plants and are the biosynthetic sites of essential compounds for normal plant growth and development (Wei

Communicated by B. Zheng.

Yuyan An and Danxuan Cheng contributed equally to this work.

Liangju Wang

wlj@njau.edu.cn

1 College of Horticulture, Nanjing Agricultural University, Street Weigang 1, Nanjing 210095, China

2 Nanjing Institute of Agricultural Sciences, Nanjing 210046, China and $\mathrm{Li}$ 2016). Therefore, improving root growth to better utilize nutrients and water in natural soil is important for the whole plant growth, and thus will be a promising approach to produce high yield crops. Understanding how to improve root growth is not only an interesting question for researchers in basic biology, but also a key for producing better performing plants.

5-Aminolevulinic acid (ALA), a key biosynthetic precursor for tetrapyrrole compounds in all living animals, plants, and microorganisms, has been proved as an environmentfriendly new plant growth regulator (Wu et al. 2019). It regulates many physiological processes of plants, including seed germination (Wang et al. 2005), guard cell movement and photosynthesis (An et al. 2016a, c), pollen germination and tube growth (An et al. 2016b), fruit coloration (Feng et al. 2016), and resistance to almost all kinds of abiotic stresses (Akram and Ashraf 2013). Increasing evidence suggests a great application potential of ALA in agriculture (Wang et al. 2003). ALA has been showed by numerous studies to be widely involved in regulating plant root growth. Hotta et al. (1997) found that ALA increased root 
growth of radish (Raphanus sativus var.) under normal conditions. Wei et al. (2012) reported that ALA significantly promoted root growth when nitrate supply was in shortage. ALA also improved root growth of cucumber (Cucumis sativus L.) (Zhen et al. 2012), Swiss chard (Beta vulgaris L. subsp. cicla) (Liu et al. 2014), rice (Oryza sativa) (Nunkaew et al. 2014), and tomato (Solanum lycopersicum) (Zhao et al. 2015) seedlings under $\mathrm{NaCl}$ stress. Moreover, ALA enhanced root growth of spring wheat (Triticum aestivum L.) (Kosar et al. 2015), oilseed rape (Brassica napus L.) (Liu et al. 2016), and Arabidopsis (An et al. 2016c) under water deficit condition. In addition, ALA also enhanced plant root growth under lead (Ali et al. 2014), cadmium (Ali et al. 2013a, b), cold (Fu et al. 2016), and waterlogging stress (An et al. 2016d). Obviously, the positive role of ALA in improving plant root growth is observed not only under appropriate condition, but also under various stressful environments. However, the exact and specific role of ALA in regulating root growth and the underlying mechanisms behind ALAregulated root growth are still largely unknown.

The root growth of plants can be regulated by various phytohormones (Lewis et al. 2011; Li et al. 2015; Mao et al. 2016; Sun et al. 2014; Xu et al. 2013; Zheng et al. 2011), most importantly auxin (Blilou et al. 2005; Petrasek et al. 2006). Auxin, which is widely and deeply investigated, is known to be involved in almost all aspects of root growth and development (Grieneisen et al. 2007). The tightly regulated distribution of auxin is crucial for its action in plants (Grieneisen et al. 2007; Petrasek et al. 2006; Sassi et al. 2012). The auxin gradients always result from local auxin biosynthesis and intercellular auxin transport which are mainly controlled by the coordination of AUX1 influx carriers and PIN efflux carriers (Ruzicka et al. 2009; Sassi et al. 2012; Zheng et al. 2011). In plants, auxin is synthesized at the apices of root and shoot and then transported to its action sites in a polar manner. Experiments using auxin transport mutants or inhibitors (Grieneisen et al. 2007), such as 2,3,5-triiodobenzoic acid (TIBA), indicate that auxin transport is crucial for generating the maximum auxin and auxin gradient. Among the auxin transport carriers, PIN efflux carriers are rate-limiting factors for auxin transport throughout the plant (Blilou et al. 2005). They localize differently in plants. PIN1, PIN3, PIN4, and PIN7 distribute mainly at the basal side of stele cells (PIN1, PIN3, and PIN7) or root stem cells (PIN4), while PIN2 always at the lateral root cap and the upper side of root epidermis (Ganguly et al. 2010). This polar localization of the PINs contributes greatly to the directional auxin flow in plants. Except the polar efflux carriers, the nonpolar AUX1 influx carriers are also required for the auxin distribution pattern at the root tips. It has been revealed that the AUX1 carriers control the distribution pattern of auxin among different tissues, whereas the PINs mainly determine the distribution of auxin within each tissue (Band et al. 2014). After being transported to the sites, where auxin functions, auxin is sensed by its receptor TRANSPORT-INHIBITOR-RESISTANT1 (TIR1) (Dharmasiri et al. 2005), mediating the auxin-responsive gene expressions through triggering the degradation of AUX/IAA proteins which decrease the activity of AUXIN RESPONSE FACTOR (ARF). Except TIR1, AUXIN BINDING PROTEIN 1 (ABP1) had ever been proposed as another potential receptor of auxin (Scherer 2011). However, Gao et al. (2015) and Enders et al. (2015) recently provided evidence that auxin signaling does not require ABP1.

In 1998, in vitro studies demonstrated that ALA showed similar characteristic to auxin in the induction of adventitious shoot and rhizogenesis (Bindu and Vivekanandan 1998). However, no further studies have been done to elucidate the relationship between ALA and auxin. Here, we hypothesized that ALA promoted root growth through modulation of auxin transport, biosynthesis, and signaling. To test our hypothesis, we first determined the specific function of ALA in regulating root growth of Arabidopsis. Then, using TIBA and auxin-related marker lines, we explored the relationship between ALA-regulated root growth and auxin transport, biosynthesis, and signaling. Our findings suggest a reciprocal interaction between ALA and auxin, providing new insights into the mechanisms underlying ALA-promoted plant root growth.

\section{Materials and methods}

\section{Plant materials and growth conditions}

Arabidopsis thaliana of wild-type (Col-0), auxin transporter reporter transgenic lines PIN1:PIN1-GFP, PIN2:PIN2-GFP (Li et al. 2015) and PIN7:PIN7-GFP (Wang et al. 2016), and auxin-responsive reporter DR5rev:GFP (Col-0, Li et al. 2015) were used in this study. Seeds of these Arabidopsis lines were sterilized by $75 \%(\mathrm{v} / \mathrm{v})$ ethanol for $45 \mathrm{~s}$ and $1 \%$ bleach for $10 \mathrm{~min}$. After being washed with sterilized water for three times, seeds were germinated and grown on $1 / 2$ Murashige and Skoog medium (MS, pH 5.8) containing $3 \%(\mathrm{w} / \mathrm{v})$ sucrose and $0.7 \%(\mathrm{w} / \mathrm{v})$ agar. The plates with seeds were placed in a culture room at $25^{\circ} \mathrm{C}$ under light of $150 \mu \mathrm{mol} \mathrm{m}^{-2} \mathrm{~s}^{-1}$ with light/dark cycles of $8 \mathrm{~h} / 16 \mathrm{~h}$.

\section{Treatments and root assay}

Three-day-old seedlings were transferred to the above described 1/2 MS medium containing different concentrations $(5-20 \mathrm{mg} / \mathrm{L})$ of ALA and $3 \mathrm{mg} / \mathrm{L} \mathrm{2,3,5-triiodoben-}$ zoic acid (TIBA) for 4-7 days. Seedlings of each treatment were then photographed and sampled separately for the 
determination of fresh weight, root length, fluorescence intensity, and gene expression.

\section{Confocal microscopy}

The fluorescence intensities in root tips of seedlings that express PIN1:PIN1-GFP, PIN2:PIN2-GFP, PIN7:PIN7$G F P$, and DR5rev:GFP were observed using a confocal laser-scanning microscope (Zeiss LSM 710) according to Li et al. (2015) and Su et al. (2016). The settings we used were $\mathrm{ex}=488 \mathrm{~nm}, \mathrm{em}=510 \sim 520 \mathrm{~nm}$, mild scanning, and frame $512 \times 512$. The fluorescence images were digitized using the Zeiss LSM image browser and processed in Adobe Photoshop. Images displayed in this study represented at least ten individual plants of each treatment. To make them comparable, we magnified all the fluorescence images same times in each experiment. To measure the fluorescence intensities, we placed a region of interest (ROI) around the root tips $(200 \mu \mathrm{m}$ from the root apex). Fluorescence intensities of membrane-localized proteins within each ROI were then quantified with the Zen software and averaged. Experiments were repeated at least three times.

\section{Quantitative real-time PCR analysis}

When 3-day-old Arabidopsis seedlings were treated for 4 days, we selected root tips (section from root meristem to the tip) of all treatments, and isolated their total RNA using RNA Isolation and Purification Kit (Tiangen, China). For cDNA synthesis, we used two micrograms of total RNA to perform reverse transcription for each sample with TransScript ${ }^{\circledR}$ One-Step gDNA Removal and cDNA Synthesis SuperMix (Transgen Biotech).

To analyze the expressions of auxin-related genes in the roots, we performed qRT-PCR in a $20 \mu \mathrm{L}$ reaction volume using chamQ SYBR qPCR Master Mix (Vazyme) according to the advices from the manufacturer. Then, we used the $2^{-\Delta \Delta C T}$ method to estimate the relative expression levels of the tested genes (Livak and Schmittgen 2001). We chose $A t U B Q$ gene as an internal control (Mao et al. 2016) and listed all the primers that were used in the study in Table 1.

\section{Statistical analysis}

All data in this study were collected from at least three independent biological replicates. Statistical analysis was carried out using the SPSS 16.0 statistical program. Data were compared with control or among treatments by analysis of variance (ANOVA) and least significant difference tests (LSD) to discriminate significant differences at $p<0.05$ or $p<0.01$.

\section{Results}

\section{ALA promotes root elongation of Arabidopsis seedlings}

To determine the relationship between ALA and plant root growth, we grew 3-day-old Arabidopsis seedlings at increasing concentrations of ALA for 4 days. We found that $5 \mathrm{mg} / \mathrm{L}$ and $10 \mathrm{mg} / \mathrm{L}$ ALA significantly promoted root length by $44.56 \%$ and $71.17 \%$, respectively (Fig. 1a, b). Consequently, $5 \mathrm{mg} / \mathrm{L}$ and $10 \mathrm{mg} / \mathrm{L}$ ALA increased root fresh weight significantly, by $20.09 \%$ and $36.07 \%$, respectively (Fig. 1c). No significant effect of $20 \mathrm{mg} / \mathrm{L}$ ALA was found on either root length or fresh weight. These results indicate that $5-10 \mathrm{mg} / \mathrm{L}$ ALA significantly promotes primary root elongation rate, improving root growth of young Arabidopsis seedlings. Since $10 \mathrm{mg} / \mathrm{L}$ ALA showed the most promotive effect, we chose it to carry out the following experiments. The time course for root length illustrated that ALA-promoted root elongation was initiated before the 2nd day (Fig. 1d). Root elongation rate of the control and ALA-treated seedlings within the experimental period was $0.34 \mathrm{~cm} /$ day and $0.50 \mathrm{~cm} /$ day, respectively. ALA promoted root elongation rate by $47.06 \%$.

\section{TIBA inhibits ALA-promoted root growth}

Auxin is the most important phytohormone that regulates plant root growth, and polar auxin transport is the most important process that mediates auxin-regulated physiological roles (Leyser 1999). To explore the relation between ALA-promoted root growth and auxin transport,
Table 1 Primer sequences for qRT-PCR analysis

\begin{tabular}{lll}
\hline Primer name & Froward primer & Reverse primer \\
\hline AtUBQ & AGAAGATCAAGCACAAGCAT & CAGATCAAGCTTCAACTCCT \\
AtYUC2 & GGATGAGACAATGGAGTATG & ATATTTCACCGCTCTTATAGG \\
AtTIR1 & CATTGTTGAGTCTTGGTCCATGTT & GTGGCTGGTCCTCGATTTGA \\
AtAUX1 & CCGAGCTGGACGGCGATGTACG & CATGTGCGGCGGCTGCAGCT \\
AtPIN1 & TACTCCGAGACCTTCCAACTACG & TCCACCGCCACCACTTCC \\
AtPIN2 & GGCGAAGAAAGCAGGAAGA & GGTGGGTACGACGGAACA \\
AtPIN7 & ACTCCTCGTCCGTCTAATCTCAC & GAAGCCATAGCACAACTCTCCTC \\
\hline
\end{tabular}



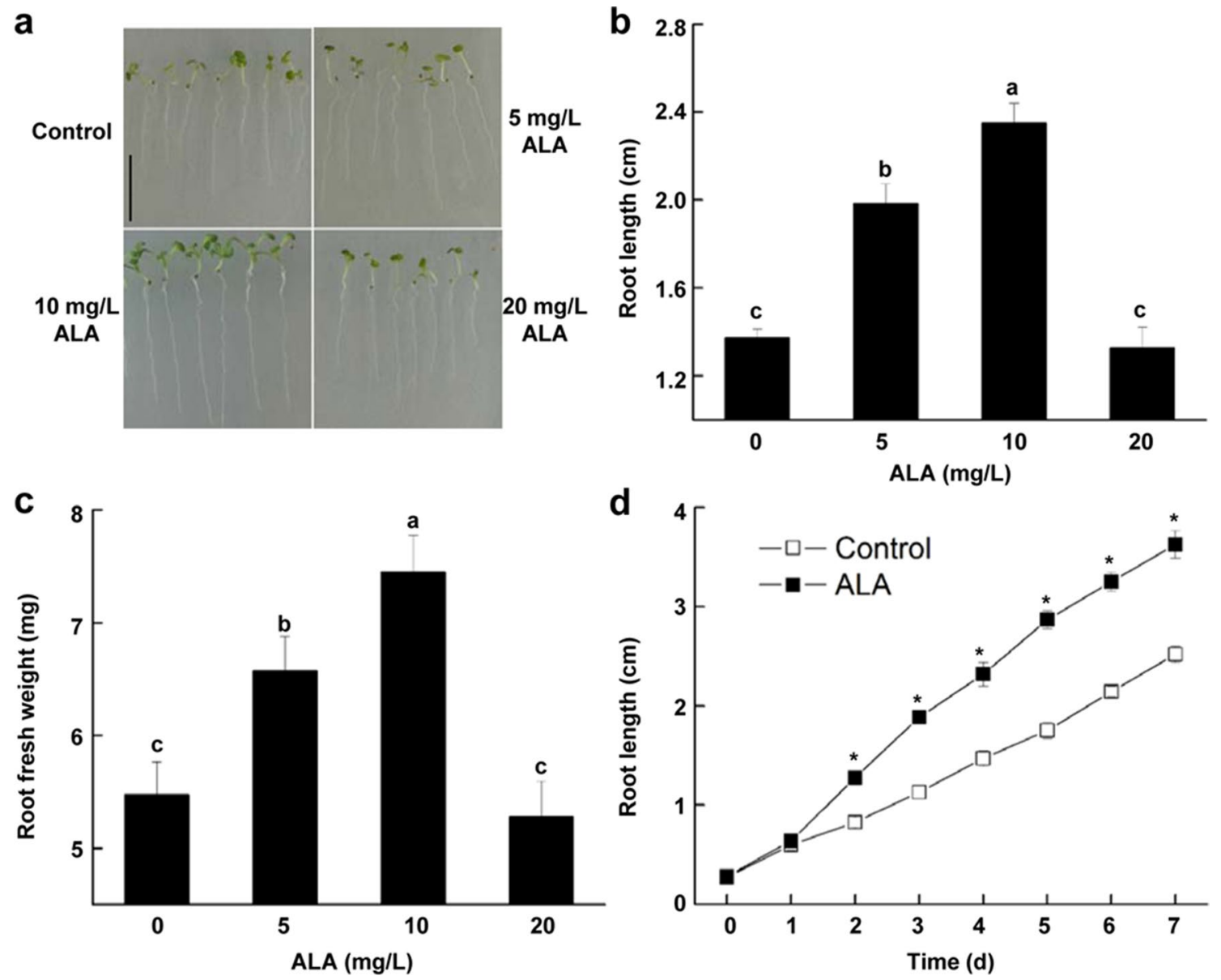

Fig. 1 ALA promotes primary root growth. Three-day-old wild-type seedlings were transferred to $1 / 2 \mathrm{MS}$ medium containing 0 (Control), 5, 10, and $20 \mathrm{mg} / \mathrm{L}$ ALA for another 4 (a-c) or 7 days (d). Digital images (a), primary root length (b), and root fresh weight (c) of seedlings treated with different concentrations of ALA for 4 days. Scale bar $=1 \mathrm{~cm}$. d Time courses of primary root length of seedlings treated

with or without $10 \mathrm{mg} / \mathrm{L}$ ALA. Values of root length or weight are the means of 45 measurements from three independent experiments (the same below). Different letters above the bars in b-c indicate significant differences at $p=0.05$ level. Asterisk in $\mathbf{d}$ indicates significant differences between treatments on the same timepoint at $p=0.05$ level

we inspected the influence of TIBA, an auxin transport inhibitor, on root growth of seedlings. Results showed that ALA alone significantly promoted root length, while TIBA alone significantly inhibited root elongation, decreasing root length by $62.53 \%$ (Fig. $2 \mathrm{a}, \mathrm{b}$ ). When ALA and TIBA applied together, both ALA's promotive effect and TIBA's suppressive effect on root length were inhibited significantly (Fig. 2a, b). The effects of ALA and TIBA on root fresh weight were accordant with those on root length (Fig. 1c). These results strongly suggest that ALA-promoted root elongation is strictly associated with auxin polar transport.

\section{ALA modulates auxin transport components}

Action of PIN proteins is rate-limiting for auxin transport, specific to auxin, and sensitive to auxin transport inhibitors (Petrasek et al. 2006). To gain more insights into the relation

between ALA and auxin transport, we investigated ALA's effects on PIN proteins using auxin transporter reporter transgenic lines PIN1:PIN1-GFP, PIN2:PIN2-GFP, and PIN7:PIN7-GFP. Results showed that ALA significantly enhanced signals of PIN1, PIN2, and PIN7 in root tips, by 59.02, 20.55, and 53.15\%, respectively (Fig. 3a, b). These results indicate that ALA upregulates the levels of these proteins in root tip. The qRT-PCR analysis showed that the relative transcript levels of these proteins were also significantly increased in ALA-treated root tips (Fig. 3c), suggesting that ALA enhances the expressions of PIN1, PIN2, and PIN7. Auxin influx carrier AUXIN RESISTANT/LIKE AUX1 is also crucial for auxin-mediated physiological processes (Band et al. 2014). We found that ALA significantly increased $A U X 1$ expression as well (Fig. 3d). These results indicate that ALA modulates several components of auxin transport. 
a

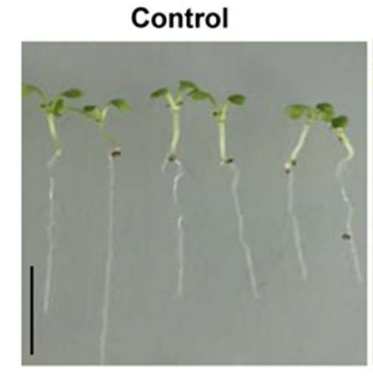

b

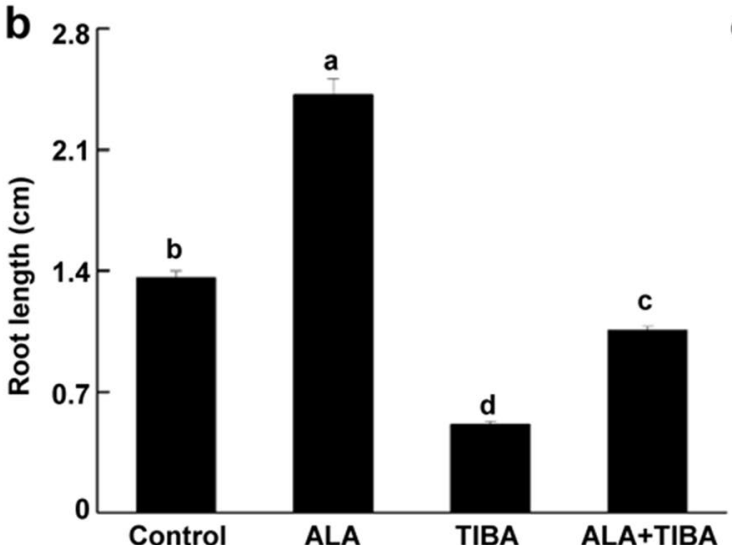

Fig. 2 TIBA inhibits ALA-promoted root growth. Three-day-old wild-type seedlings were transferred to $1 / 2 \mathrm{MS}$ medium alone (control) or containing $10 \mathrm{mg} / \mathrm{L}$ ALA, $3 \mathrm{mg} / \mathrm{L}$ TIBA, or $10 \mathrm{mg} / \mathrm{L}$ $\mathrm{ALA}+3 \mathrm{mg} / \mathrm{L}$ TIBA for another 4 days. Digital images (a), primary

To further confirm its role in regulating auxin transport components, we investigated whether ALA could compensate for the effects of TIBA on auxin transport components. TIBA significantly decreased the signals of PIN1, PIN2, and PIN7 in root tips (Fig. 4a, b), reducing the levels of these proteins. The qRT-PCR analysis showed that TIBA also significantly decreased the relative transcript levels of PINI, PIN2, and PIN7 in root tips (Fig. 4c). However, when TIBA was applied together with ALA, its effects on PIN1, PIN2, and PIN7 were significantly inhibited both at protein and transcriptional levels (Fig. 4a-c). ALA recovered PIN2 and PIN7 to the corresponding control level, both at protein and transcriptional levels (Fig. 4b, c). Moreover, ALA's effects on PIN2 and PIN7 proteins correlated well with its effects on PIN2 and PIN7 transcription. While for PIN1, ALA's effects on the transcript and protein levels did not correlate well with each other. ALA recovered PIN1 to the control level at protein level but not at transcript level. TIBA also significantly decreased $A U X 1$ expression (Fig. 4d). Different from its effects on auxin efflux carriers, ALA did not inhibit TIBA-decreased $A U X 1$ expression. These results confirm the regulation of ALA on anxin transport components, indicating that ALA may improve polar auxin transport through modulation of auxin transport components, especially auxin efflux carriers.
TIBA

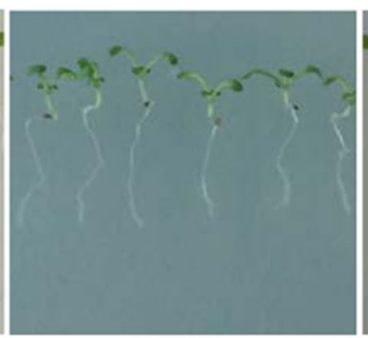

ALA+TIBA

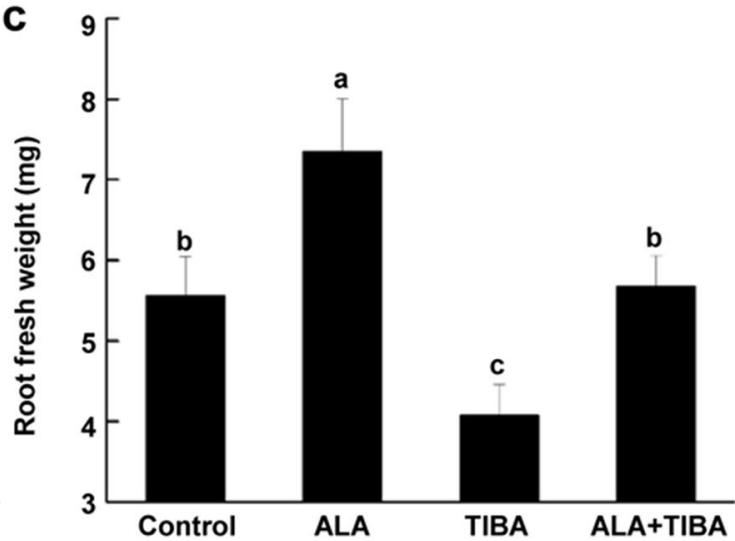

root length (b), and root fresh weight (c) of seedlings under different treatments for 4 days. Scale bar $=1 \mathrm{~cm}$. Different letters above the bars indicate significant differences at $p=0.05$ level

\section{ALA improves auxin accumulation and response in roots}

Auxin gradients are crucial for many plant developmental processes regulated by auxin, including primary root growth. In Arabidopsis, DR5 promoter is a reporter for auxin distribution and auxin-signaling responses (Wang et al. 2016). Therefore, we used DR5rev:GFP transgenic line to gain more information on the role of ALA in auxin-signaling responses. In control roots, DR5 signal mainly focused on the quiescent zones and the surrounding columella cells of root cap. ALA treatment resulted in enhanced DR5 signal not only in the columella cells of root caps, but also in the elongation zone (Fig. 5a, b). Moreover, ALA also increased DR5 signal in root stele (Fig. 5a). These data indicate that ALA stimulates auxin accumulation in root tip and enhances the response to auxin. Compared with the control, TIBA alone significantly reduced DR5 signal (Fig. 5c, d). However, when applied together with ALA, TIBA's effect on DR5 signal was totally reversed (Fig. 5c, d), further confirming ALA's positive role in auxin accumulation and response.

Besides auxin transport, auxin accumulation may also result from the upregulation of its biosynthesis. To determine whether ALA-induced auxin accumulation is associated with auxin biosynthesis, we investigated how ALA 
a

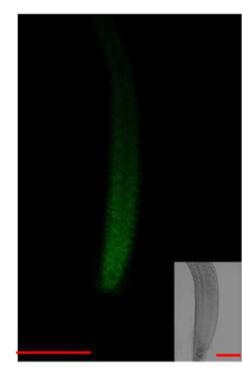

Control
PIN1::PIN1-GFP

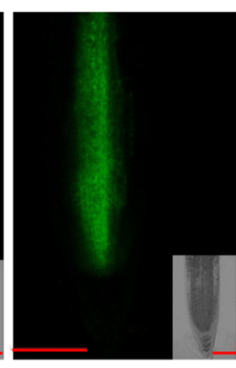

ALA

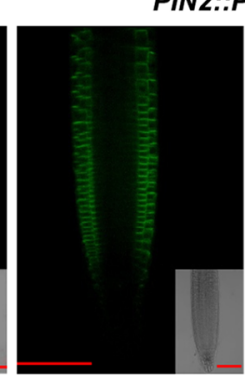

Control
PIN2::PIN2-GFP

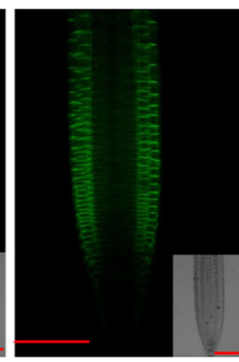

ALA

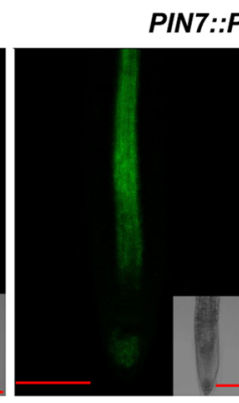

Control
PIN7::PIN7-GFP

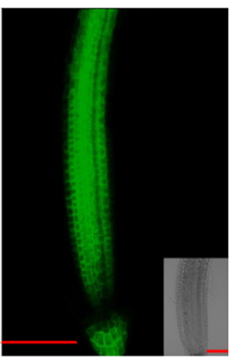

ALA

b
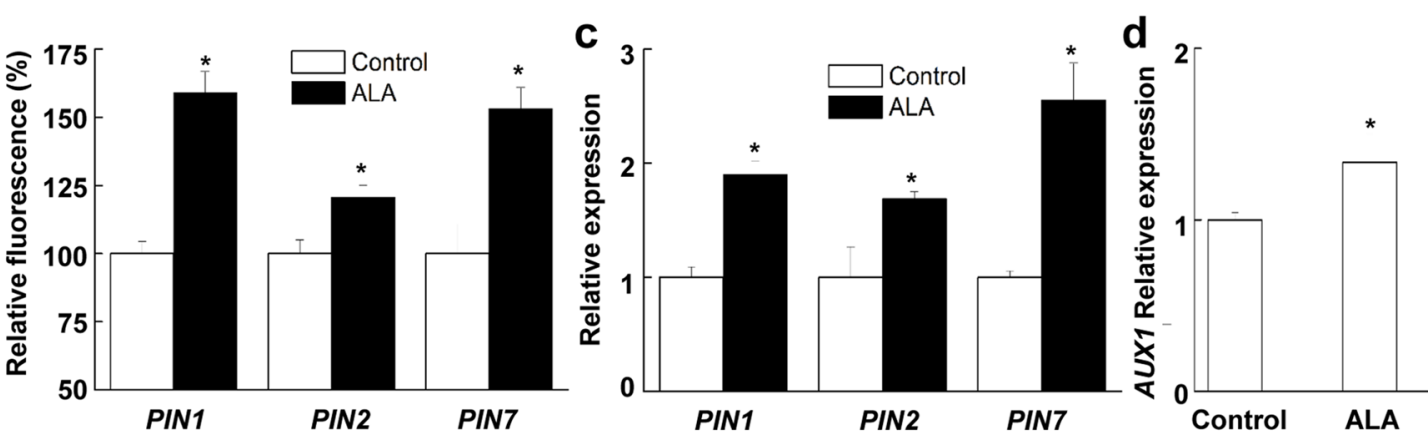

Fig. 3 ALA modulates auxin transport components. Three-dayold seedlings expressing PIN1:PIN1-GFP, PIN2:PIN2-GFP, and PIN7:PIN7-GFP were transferred to $1 / 2 \mathrm{MS}$ medium with or without $10 \mathrm{mg} / \mathrm{L}$ ALA for another 4 days. Signals of PIN1, PIN2, and PIN7 were observed using a confocal laser-scanning microscope (CLSM). a Fluorescence images representing the abundance of PIN proteins in roots and the images in the lower right corners are in bright field. Scale bar $=50 \mu \mathrm{m}$. b GFP fluorescence intensity of PIN1, PIN2, and PIN7 as in a. The fluorescence intensity of the control was set to $100 \%$. Values are the means of 30 measurements \pm SE from three independent experiments. c qRT-PCR analysis of PIN1, PIN2, and PIN7 in root tips. d qRT-PCR analysis of AUX1 in root tips. In $\mathbf{c}$ and d, the expression levels in control roots were set to 1 . Values represent nine measurements from three independent experiments. Asterisk in b-d indicates significant differences between control and ALA treatment at $p=0.05$ level and TIBA affect the expression of $Y U C 2$, one important auxin biosynthetic genes. Results showed that ALA significantly enhanced the expression of YUC2 (Fig. 5e). TIBA alone did not significantly affect, but slightly increased YUC2 expression (Fig. 5f). This could be due to that plants produce more auxin to counteract the inhibition of auxin transport. ALA and TIBA together enhanced $Y U C 2$ expression to the same extent as TIBA alone (Fig. 5f), suggesting that the inhibition of TIBA-decreased auxin accumulation and response by ALA (Fig. 5c, d) may not mainly result from ALA-regulated auxin biosynthesis.

\section{ALA does not affect TIR1 expression}

The above results proved that ALA affected auxin transport and biosynthesis; next, we investigated whether ALA modulated auxin signaling in the roots. Results showed that ALA, with or without TIBA, did not affect the expression of TIRI (Fig. 6), indicating that ALA may not affect root growth mainly through regulation of auxin receptor at the transcript level.

\section{Discussion}

ALA is not only an essential precursor in tetrapyrrole biosynthesis; increasing studies indicate that it is also a vital new plant growth regulator (Wu et al. 2019). ALA regulates many important physiological processes, such as improving seed germination (Wang et al. 2005), improving plant photosynthesis and growth (Hotta et al. 1997), and increasing plant tolerance to nearly all of the environmental stresses (Akram and Ashraf 2013; Akram et al. 2018). Recently, we showed evidence of ALA's new physiological roles in regulating pollen tube growth and stomatal movement (An et al. 2016a, b, c). The multiple effects of ALA on the key agricultural traits of plants suggest a great application potential of ALA in agriculture (Wu et al. 2019). Due to its various physiological roles and its natural, environmentally friend characteristics, increasing attention has been drawn on ALA and the mechanisms behind ALA's functions. Previous studies have indicated that ALA promotes plant growth including root biomass in both normal and stressful conditions (Ali et al. 2013b, 2014; An et al. 2016d; Hotta et al. 1997; Liu et al. 2014, 2016; Wei et al. 2012; Zhen et al. 2012; 
a
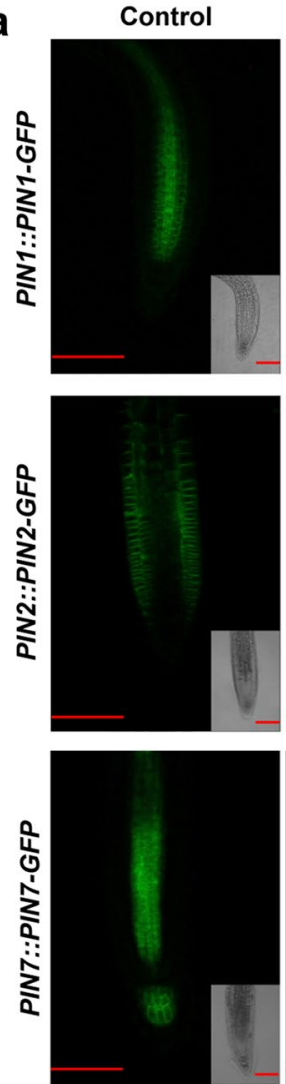

TIBA
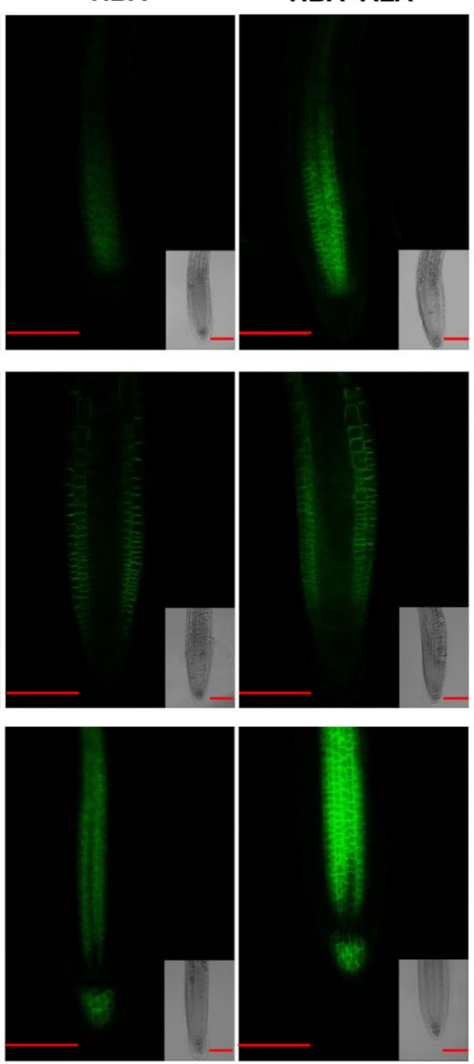

TIBA+ALA
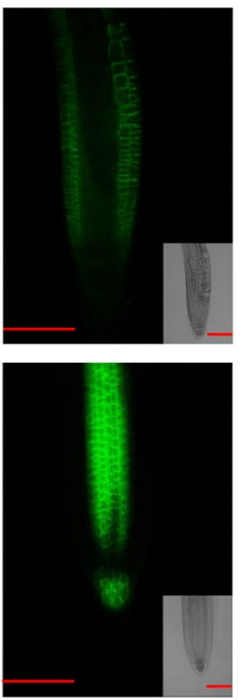

b

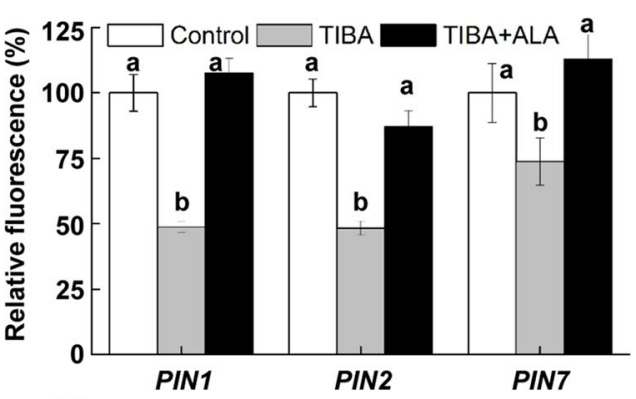

C

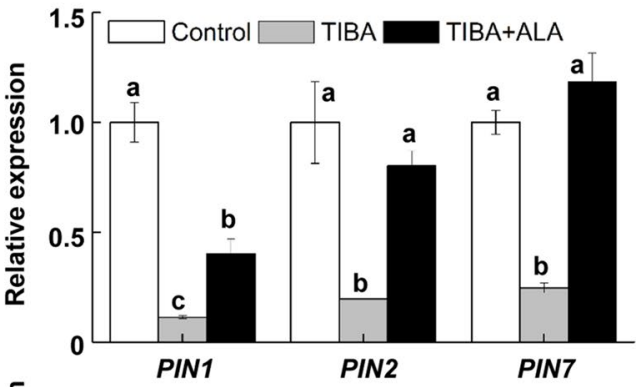

d

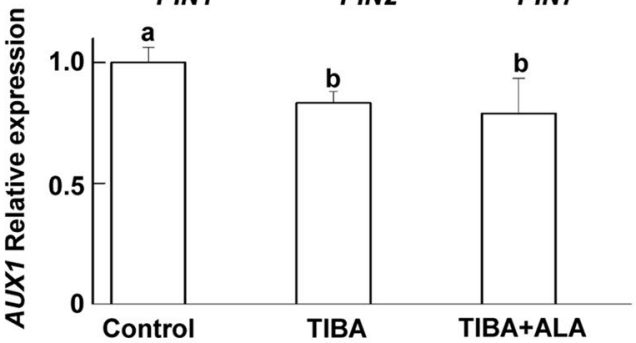

Fig. 4 ALA inhibits TIBA-regulated auxin transport in roots. Threeday-old seedlings expressing PIN1:PIN1-GFP, PIN2:PIN2-GFP, and PIN7:PIN7-GFP were transferred to $1 / 2 \mathrm{MS}$ medium alone, or containing $3 \mathrm{mg} / \mathrm{L}$ TIBA with or without $10 \mathrm{mg} / \mathrm{L}$ ALA for another 4 days. a Fluorescence images representing the abundance of PIN proteins in roots and the images in the lower right corners are in bright field. Scale bar $=50 \mu \mathrm{m}$. b GFP fluorescence intensity of PIN1,

Zhao et al. 2015). To date, however, little specific information is available on ALA-regulated root growth and its underlying mechanisms. In this study, we showed that ALA at $5-10 \mathrm{mg} / \mathrm{L}$ significantly increased primary root length of Arabidopsis seedlings (Fig. 1). Because the Arabidopsis seedlings in this study were not allowed to grow older than 10 days, we did not observe the effects of ALA on the initiation and growth of lateral roots. However, our another study on strawberry showed that $10 \mathrm{mg} / \mathrm{L}$ ALA improved not only adventitious root, but also lateral root elongation, while $20 \mathrm{mg} / \mathrm{L}$ ALA significantly inhibited adventitious root elongation, but promoted the initiation of lateral roots (data not published yet). These results indicate that ALA affects root growth of plants in a concentration-dependent manner. Low concentrations of ALA improve root elongation, while higher concentrations may not improve root length, but stimulate initiation of lateral roots.

Auxin, the most important plant hormone regulating root growth, may also modulate root growth positively or
PIN2, and PIN7 as in a. The fluorescence intensity of the control was set to $100 \%$. Values are the means of 30 measurements \pm SE from three independent experiments. c qRT-PCR analysis of PIN1, PIN2, and PIN7 in root tips. d qRT-PCR analysis of AUX1 in root tips. Different letters on the bars in $\mathbf{b}-\mathbf{d}$ indicate significant differences between treatments at $p=0.05$ level

negatively, depending on its concentration ( $\mathrm{Li}$ et al. 2015). Therefore, in regulating root growth, the effect of ALA is mimic to that of auxin. Actually, Bindu and Vivekanandan (1998) have reported that ALA showed similar characteristic to auxin in the induction of callusing and rhizogenesis. However, no more research has been performed to study the relationship between auxin and ALA, and the link between auxin and ALA-regulated root growth is entirely unknown. Here, we showed that ALA regulated root growth probably by modulating auxin transport (Figs. 2, 3, 4) and biosynthesis (Fig. 5), indicating an interaction between ALA and auxin in regulating plant root growth.

The function of auxin in regulating root growth of plants is mostly dependent on its accumulation and distribution in the root apex, which are mainly controlled by auxin biosynthesis and polar transport ( $\mathrm{Li}$ et al. 2015; Wang et al. 2016). Directional auxin transport is especially important for distribution of auxin in plant root tips (Grieneisen et al. 2007). In recent years, increasing molecular data have been 


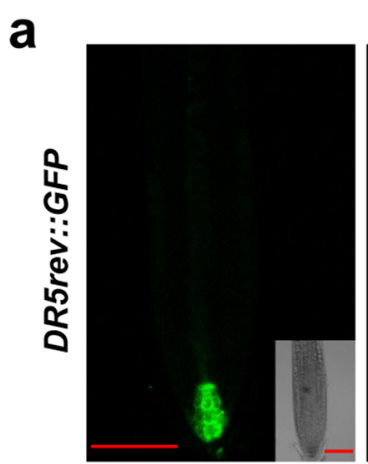

Control

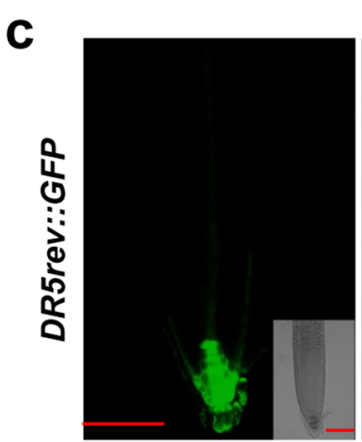

Control

e

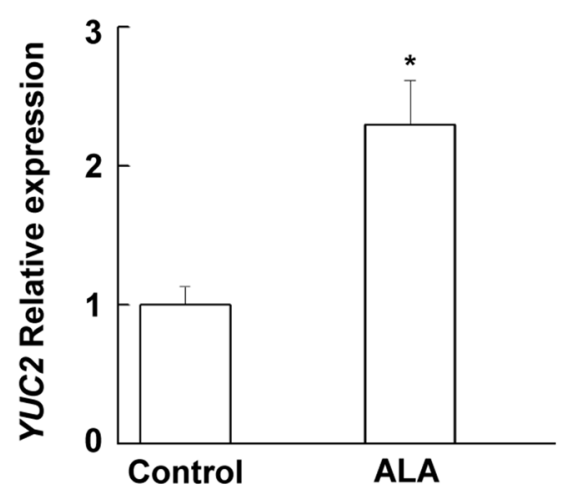

b

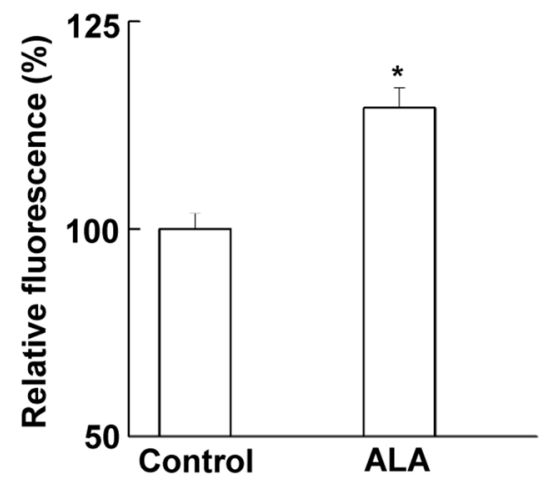

ALA

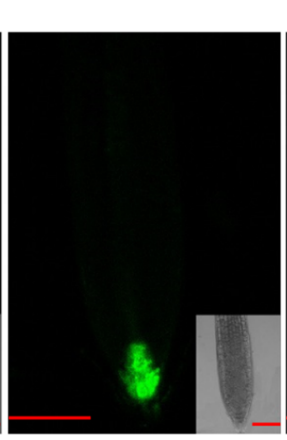

TIBA

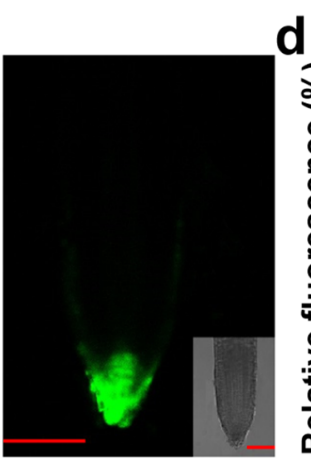

TIBA+ALA

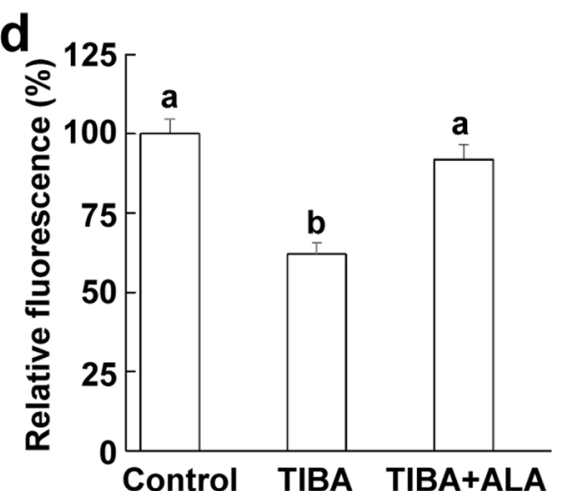

Control TIBA TIBA+ALA f

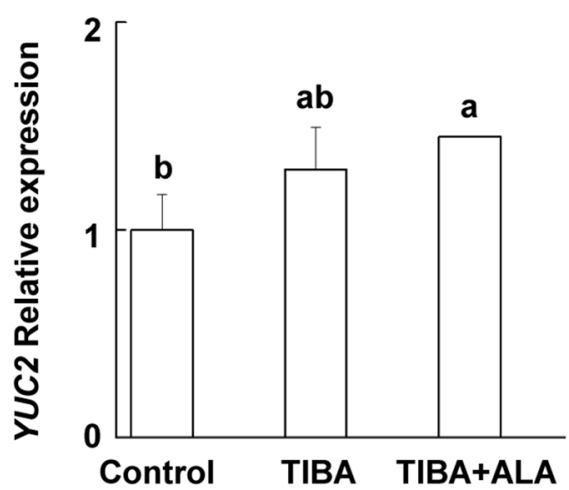

Fig. 5 ALA enhances auxin accumulation and response in roots. Three-day-old seedlings expressing DR5rev:GFP were transferred to $1 / 2 \mathrm{MS}$ medium with or without $10 \mathrm{mg} / \mathrm{L}$ ALA for another 4 days. Signals of DR5 were observed using a CLSM. a Fluorescence images representing the abundance of DR5 proteins in roots and the images in the lower right corners are in bright field. Scale bar $=50 \mu \mathrm{m}$. b GFP fluorescence intensity of DR5 as in a. The fluorescence intensity of the control was set to $100 \%$. e qRT-PCR analysis of YUC2 in root tips. Asterisk in $\mathbf{b}$ and $\mathbf{e}$ indicates significant differences between control and ALA treatment at $p=0.05$ level. Three-day-old seedlings expressing DR5rev: GFP were transferred to $1 / 2 \mathrm{MS}$ medium alone, or containing $3 \mathrm{mg} / \mathrm{L}$ TIBA with or without $10 \mathrm{mg} / \mathrm{L}$ ALA for another 4 days. Signals of DR5 were observed using a CLSM. c Fluorescence images representing the abundance of DR5 proteins in roots and the images in the lower right corners are in bright field. Scale bar $=50 \mu \mathrm{m}$. d GFP fluorescence intensity of DR5 as in c. The fluorescence intensity of the control was set to $100 \%$. f qRT-PCR analysis of YUC2 in root tips. In e and $\mathbf{f}$, the expression levels in control roots were set to 1 . The same letters on the bars in $\mathrm{d}$ and $\mathrm{f}$ indicate no significant differences between treatments at $p=0.05$ level. Values are the means of $n$ measurements from three independent experiments $(n=30$ in $\mathbf{b}$ and $\mathbf{d}, n=9$ in $\mathbf{e}$ and $\mathbf{f})$ generated on the mechanisms behind auxin transport and its regulation (Vieten et al. 2007), providing many valuable chemicals and plant materials for the following research. In this study, TIBA, an auxin transport inhibitor, inhibited ALA-induced root elongation (Fig. 2), indicating that a close relationship between auxin transport and ALA-regulated root growth. PIN proteins, the auxin efflux carriers, play crucial roles in regulating the direction of auxin flow, thus they are very important for auxin-regulated root growth (Blilou et al. 2005). PIN1 and PIN7 mainly regulate acropetal auxin transport, while PIN2 specifically influences the basipetal transport of auxin (Blilou et al. 2005; Ruzicka et al. 2007). 


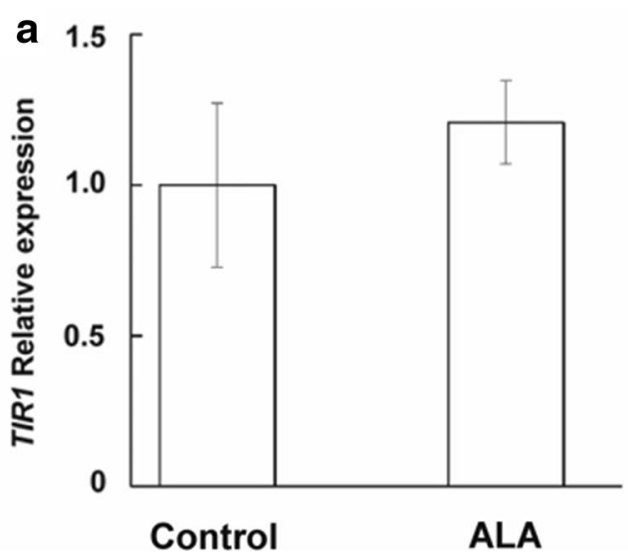

Fig. 6 Effects of ALA on transcript levels of TIR1. a Three-dayold wild-type seedlings were transferred to $1 / 2 \mathrm{MS}$ medium with or without $10 \mathrm{mg} / \mathrm{L}$ ALA for another 4 days. b Three-day-old wild-type seedlings were transferred to $1 / 2 \mathrm{MS}$ medium alone, or containing

Here, using the auxin transporter reporter transgenic lines PIN1:PIN1-GFP, PIN2:PIN2-GFP, and PIN7:PIN7-GFP, we found that ALA significantly increased the protein levels of PIN1, PIN2, and PIN7 (Fig. 3a, b) and inhibited the negative effects of TIBA on these PINs (Fig. 4a, b). These results indicate that ALA not only improves acropetal auxin transport, but also increases basipetal transport of auxin. The PIN-mediated regulation of auxin transport and distribution controls not only cell division, but also cell elongation (Blilou et al. 2005), two major processes determining root growth. Therefore, the improved levels of PINs induced by ALA may contribute largely to ALA-stimulated root growth. The qRT-PCR experiment showed that ALA enhanced the expression of PIN1, PIN2, and PIN7 (Fig. 3c) and inhibited TIBA-suppressed expression of PIN1, PIN2, and PIN7 (Fig. 4c). The changes in transcript levels of PIN2 and PIN7 correlated very well with their protein levels in this study, indicating that ALA enhances accumulation of PIN2 and PIN7 proteins mainly through regulating their transcription. ALA recovered PIN1 completely at protein level, but partially at transcript level, indicating that ALA regulates protein PIN1 at both transcriptional and post-transcription levels. Except PINs, the influx carrier AUX1 also regulates auxin distribution in the root (Band et al. 2014). We did not analyze AUX1 distributions in the root. However, qRTPCR analysis showed that ALA enhanced AUXI expression under normal condition (Fig. 3d), but did not inhibit TIBAdecreased AUX1 expression (Fig. 4d). These results indicate that ALA regulates auxin transport mainly through influencing PIN efflux auxin carriers instead of influx protein AUX1. Whether ALA affects the members of the multidrugresistant $P$-glycoprotein, another kind of auxin transporters (Ruzicka et al. 2009; Sassi et al. 2012; Zheng et al. 2011) needs to be further studied.

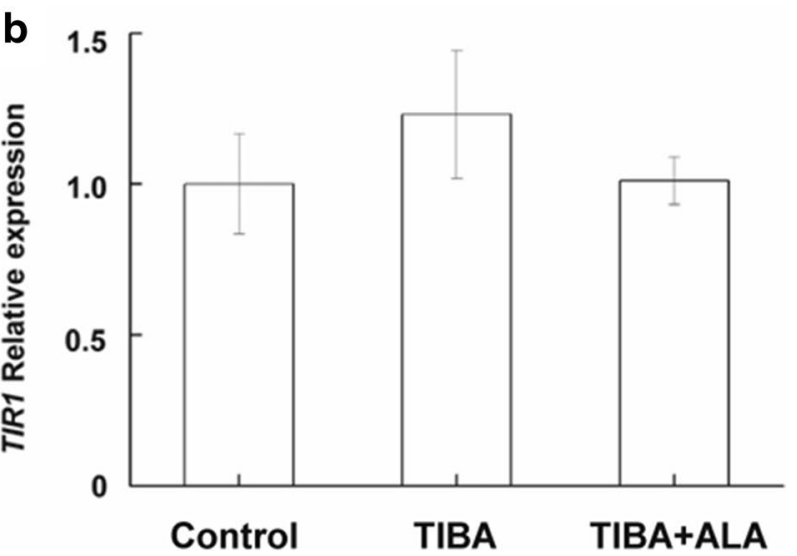

$3 \mathrm{mg} / \mathrm{L}$ TIBA with or without $10 \mathrm{mg} / \mathrm{L}$ ALA for another 4 days. Total RNA was isolated from root tips for qRT-PCR analysis of TIR1. The expression levels in control roots were set to 1 . Values are the averages of nine measurements from three independent experiments

The process of root organogenesis is controlled by auxin and maintaining root apical auxin maximum is important for regulating primary root growth (Koprivova et al. 2010). Using transgenic Arabidopsis lines that expressing GFP under the control of auxin-responsive DR5 promoter $(\mathrm{Li}$ et al. 2015), we observed that ALA strengthened auxindependent reporter expression in roots (Fig. 5a-d). These results indicate that ALA stimulates the auxin accumulation in the root tip, resulting in the better maintenance of the apical auxin maximum. The accumulation of auxin in root tips may be caused by the activation of its synthesis or transport (Koprivova et al. 2010). In the present study, the improvement of auxin transport by ALA is consistent with the expression pattern of DR5, suggesting that ALAimproved auxin transport is a major cause of auxin accumulation. $Y U C 2$ is one of the key genes of auxin biosynthesis (Wang et al. 2016). ALA significantly enhanced the expression of $Y U C 2$. However, compared with TIBA alone, TIBA and ALA together did not enhance $Y U C 2$ expression. This result is not consistent with the changing expression pattern of DR5 (Fig. 5c, d). These data indicate that ALAinduced auxin synthesis may not be the major reason for auxin accumulation in root tips. However, YUC1, YUC2, $Y U C 5, Y U C 6$, and TAR2 are also the key genes of auxin biosynthesis (Wang et al. 2016), and we only determined the expression of one of them. Therefore, the exact impact of ALA on auxin biosynthesis needs to be further studied.

In plants, the changing auxin levels will be sensed by auxin perception machinery and result in related changes in gene expressions, which then contributes to the modulation of elongation growth (Ljung 2013; Li et al. 2015). TIR1 is a recognized auxin receptor (Dharmasiri et al. 2005; Scherer 2011). In this study, we found that ALA did not impact the relative expression of TIRI in root tips (Fig. 6). This result 
indicates that ALA may not mediate auxin responses through TIR1-mediated auxin signaling at the transcript level. ALA's effect on auxin signaling will be further studied at the protein level in the near future.

In summary, our data demonstrate the effects of ALA on root growth, and indicate the involvement of auxin responses in ALA-regulated root growth. Some evidence for ALAimproved auxin transport and biosynthesis is presented, providing new insights into the functional mechanisms of ALA. The direct link between ALA-regulated root growth and auxin will be further deeply studied in the future.

Author contribution statement YA and LW made the major contributions in designing the experiments, writing, and revising manuscript. YA, DC, and ZR performed all the experiments. YS and QT supplied plant materials and revised the manuscript. All authors read and approved the manuscript.

Acknowledgements We gratefully thank Dr. Wei Xuan from Huazhong Agricultural University and Dr. Jian Xu from National University of Singapore for supplying seeds of Arabidopsis transgenic lines DR5rev:GFP, PIN1:PIN1-GFP, PIN2:PIN2-GFP and PIN7:PIN7$G F P$. This work was financially supported by the National Natural Science Foundation of China (Grant Nos. 31772253, 31401820).

Open Access This article is distributed under the terms of the Creative Commons Attribution 4.0 International License (http://creativeco mmons.org/licenses/by/4.0/), which permits unrestricted use, distribution, and reproduction in any medium, provided you give appropriate credit to the original author(s) and the source, provide a link to the Creative Commons license, and indicate if changes were made.

\section{References}

Akram NA, Ashraf M (2013) Regulation in plant stress tolerance by a potential plant growth regulator, 5-aminolevulinic acid. J Plant Growth Regul 32:663-679. https://doi.org/10.1007/s0034 4-013-9325-9

Akram NA, Iqbal M, Muhammad A, Ashraf M, Al-Qurainy F, Shafiq $S$ (2018) Aminolevulinic acid and nitric oxide regulate oxidative defense and secondary metabolisms in canola (Brassica napus L.) under drought stress. Protoplasma 255:163-174. https://doi. org/10.1007/s00709-017-1140-x

Ali B, Huang CR, Qi ZY, Ali S, Daud MK, Geng XX, Liu HB, Zhou WJ (2013a) 5-Aminolevulinic acid ameliorates cadmium-induced morphological, biochemical, and ultrastructural changes in seedlings of oilseed rape. Env Sci Pollut Res 20:7256-7267. https:// doi.org/10.1007/s11356-013-1735-5

Ali B, Wang B, Ali S, Ghani MA, Hayat MT, Yang C, Xu L, Zhou WJ (2013b) 5-Aminolevulinic acid ameliorates the growth, photosynthetic gas exchange capacity, and ultrastructural changes under cadmium stress in Brassica napus L. J Plant Growth Regul 32:604-614. https://doi.org/10.1007/s00344-013-9328-6

Ali B, Xu X, Gill RA, Yang S, Ali S, Tahir M, Zhou WJ (2014) Promotive role of 5-aminolevulinic acid on mineral nutrients and antioxidative defense system under lead toxicity in Brassica napus. Ind Crops Pro 52:617-626. https://doi.org/10.1016/j. indcrop.2013.11.033

An YY, Feng XX, Liu LB, Xiong LJ, Wang LJ (2016a) ALA-induced flavonols accumulation in guard cells is involved in scavenging $\mathrm{H}_{2} \mathrm{O}_{2}$ and inhibiting stomatal closure in Arabidopsis cotyledons. Front Plant Sci 7:1713. https://doi.org/10.3389/fpls.2016.01713

An YY, Li J, Duan CH, Liu LB, Sun YP, Cao RX, Wang LJ (2016b) 5-Aminolevulinic acid thins pear fruits by inhibiting pollen tube growth via $\mathrm{Ca}^{2+}$-ATPase-mediated $\mathrm{Ca}^{2+}$ efflux. Front Plant Sci 7:121. https://doi.org/10.3389/fpls.2016.00121

An YY, Liu LB, Chen LH, Wang LJ (2016c) ALA inhibits ABAinduced stomatal closure via reducing $\mathrm{H}_{2} \mathrm{O}_{2}$ and $\mathrm{Ca}^{2+}$ levels in guard cells. Front Plant Sci 7:482. https://doi.org/10.3389/ fpls.2016.00482

An YY, Qi L, Wang LJ (2016d) ALA pretreatment improves waterlogging tolerance of fig plants. PLoS One 11:0147202. https:// doi.org/10.1371/journal.pone.0147202

Band LR, Wells DM, Fozard JA, Ghetiu T, French AP, Pound MP, Wilson MH, Yu L, Li WD, Hijazi HI, Oh J, Pearce SP, PerezAmador MA, Yun J, Kramer E, Alonso JM, Godin C, Vernoux T, Hodgman TC, Pridmore TP, Swarup R, King JR, Bennett MJ (2014) Systems analysis of auxin transport in the Arabidopsis root apex. Plant Cell 26:862-875. https://doi.org/10.1105/ tpc.113.119495

Bindu RC, Vivekanandan M (1998) Hormonal activities of 5-aminolevulinic acid in callus induction and micropropagation. Plant Growth Regul 26:15-18. https://doi.org/10.1023/A:1006098005 335

Blilou I, Xu J, Wildwater M, Willemsen V, Paponov I, Friml J, Heidstra R, Aida M, Palme K, Scheres B (2005) The PIN auxin efflux facilitator network controls growth and patterning in Arabidopsis roots. Nature 433:39-44. https://doi.org/10.1038/nature03184

Dharmasiri N, Dharmasiri S, Estelle M (2005) The F-box protein TIR1 is an auxin receptor. Nature 435:441-445. https://doi.org/10.1038/ nature 03543

Enders TA, Oh S, Yang Z, Montgomery BL, Strader LC (2015) Genome sequencing of Arabidopsis abpl-5 reveals second-site mutations that may affect phenotypes. Plant Cell 27:1820-1826. https://doi.org/10.1105/tpc.15.00214

Feng XX, An YY, Zheng J, Sun M, Wang LJ (2016) Proteomics and SSH analyses of ALA-promoted fruit coloration and evidence for the involvement of a MADS-Box gene, MdMADS1. Front Plant Sci 7:1615. https://doi.org/10.3389/fpls.2016.01615

Fu JJ, Chu XT, Sun YF, Xu YF, Hu TM (2016) Involvement of nitric oxide in 5-aminolevulinic acid-induced antioxidant defense in roots of Elymus nutans exposed to cold stress. Biol Plant 60:585594. https://doi.org/10.1007/s10535-016-0635-1

Ganguly A, Lee SH, Cho M, Lee OR, Yoo H, Cho HT (2010) Differential auxin-transporting activities of PIN-FORMED proteins in Arabidopsis root hair cells. Plant Physiol 153:1046-1061. https ://doi.org/10.1104/pp.110.156505

Gao Y, Zhang Y, Zhang D, Dai X, Estelle M, Zhao Y (2015) Auxin binding protein $1(\mathrm{ABP} 1)$ is not required for either auxin signaling or Arabidopsis development. Proc Natl Acad Sci USA 112:22752280. https://doi.org/10.1073/pnas.1500365112

Grieneisen VA, Xu J, Maree AFM, Hogeweg P, Scheres B (2007) Auxin transport is sufficient to generate a maximum and gradient guiding root growth. Nature 449:1008-1013. https://doi. org/10.1038/nature06215

Hotta Y, Tanaka T, Takaoka H, Takeuchi Y, Konnai M (1997) Promotive effects of 5-aminolevulinic acid on the yield of several crops. Plant Growth Regul 22:109-114. https://doi.org/10.1023/a:10058 83930727

Koprivova A, Mugford ST, Kopriva S (2010) Arabidopsis root growth dependence on glutathione is linked to auxin transport. Plant Cell Rep 29:1157-1167. https://doi.org/10.1007/s00299-010-0902-0 
Kosar F, Akram NA, Ashraf M (2015) Exogenously-applied 5-aminolevulinic acid modulates some key physiological characteristics and antioxidative defense system in spring wheat (Triticum aestivum L.) seedlings under water stress. S Afr J Bot 96:71-77. https://doi. org/10.1016/j.sajb.2014

Lewis DR, Negi S, Sukumar P, Muday GK (2011) Ethylene inhibits lateral root development, increases IAA transport and expression of PIN3 and PIN7 auxin efflux carriers. Development 138:3485-3495. https://doi.org/10.1242/dev.065102

Leyser O (1999) Plant hormones: ins and outs of auxin transport. Curr Biol 9:R8-R10. https://doi.org/10.1016/S0960-9822(99)80033-5

Li GJ, Zhu CH, Gan LJ, Ng D, Xia K (2015) GA 3 enhances root responsiveness to exogenous IAA by modulating auxin transport and signaling in Arabidopsis. Plant Cell Rep 34:483-494. https://doi. org/10.1007/s00299-014-1728-y

Liu LY, Nguyen NT, Ueda A, Saneoka H (2014) Effects of 5-aminolevulinic acid on Swiss chard (Beta vulgaris L. subsp cicla) seedling growth under saline conditions. Plant Growth Regul 74:219-228. https://doi.org/10.1007/s10725-014-9913-0

Liu D, Kong DD, Fu XK, Ali B, Xu L, Zhou WJ (2016) Influence of exogenous 5-aminolevulinic acid on chlorophyll synthesis and related gene expression in oilseed rape de-etiolated cotyledons under water-deficit stress. Photosynthetica 54:468-474. https://doi. org/10.1007/s11099-016-0197-7

Livak KJ, Schmittgen TD (2001) Analysis of relative gene expression data using real-time quantitative PCR and the $2^{-\Delta \Delta C T}$ method. Methods 25:402-408. https://doi.org/10.1006/meth.2001.1262

Ljung K (2013) Auxin metabolism and homeostasis during plant development. Development 140:943-950. https://doi.org/10.1242/dev.086363

Mao JL, Miao ZQ, Wang Z, Yu LH, Cai XT, Xiang CB (2016) Arabidopsis ERF1 mediates cross-talk between ethylene and auxin biosynthesis during primary root elongation by regulating ASA1 Expression. PLoS Genet 12:e1006076. https://doi.org/10.1371/ journal.pgen. 1005760

Nunkaew T, Kantachote D, Kanzaki H, Nitoda T, Ritchie RJ (2014) Effects of 5-aminolevulinic acid (ALA)-containing supernatants from selected Rhodopseudomonas palustris strains on rice growth under $\mathrm{NaCl}$ stress, with mediating effects on chlorophyll, photosynthetic electron transport and antioxidative enzymes. Electron J Biotechn 17:19-26. https://doi.org/10.1016/j.ejbt.2013.12.004

Petrasek J, Mravec J, Bouchard R, Blakeslee JJ, Abas M, Seifertova D, Wisniewska J, Tadele Z, Kubes M, Covanova M, Dhonukshe P, Skupa P, Benkova E, Perry L, Krecek P, Lee OR, Fink GR, Geisler M, Murphy AS, Luschnig C, Zazimalova E, Friml J (2006) PIN proteins perform a rate-limiting function in cellular auxin efflux. Science 312:914-918. https://doi.org/10.1126/science.1123542

Ruzicka K, Ljung K, Vanneste S, Podhorska R, Beeckman T, Friml J, Benkova E (2007) Ethylene regulates root growth through effects on auxin biosynthesis and transport-dependent auxin distribution. Plant Cell 19:2197-2212. https://doi.org/10.1105/tpc.107.052126

Ruzicka K, Simaskova M, Duclercq J, Petrasek J, Zazimalova E, Simon S, Friml J, Van Montagu MCE, Benkova E (2009) Cytokinin regulates root meristem activity via modulation of the polar auxin transport. Proc Natl Acad Sci USA 106:4284-4289. https://doi.org/10.1073/ pnas.0900060106

Sassi M, Lu YF, Zhang YH, Wang J, Dhonukshe P, Blilou I, Dai MQ, Li J, Gong XM, Jaillais Y, Yu XH, Traas J, Ruberti I, Wang HY, Scheres B, Vernoux T, Xu J (2012) COP1 mediates the coordination of root and shoot growth by light through modulation of PIN1and PIN2-dependent auxin transport in Arabidopsis. Development 139:3402-3412. https://doi.org/10.1242/dev.078212

Scherer GFE (2011) AUXIN-BINDING-PROTEIN1, the second auxin receptor: what is the significance of a two-receptor concept in plant signal transduction? J Exp Bot 62:3339-3357. https://doi. org/10.1093/jxb/err033
Slovak R, Ogura T, Satbhai SB, Ristova D, Busch W (2016) Genetic control of root growth: from genes to networks. Ann Bot 117:9-24. https://doi.org/10.1093/aob/mcv160

Su C, Liu L, Liu HP, Ferguson BJ, Zou YM, Zhao YK, Wang T, Wang $\mathrm{YN}, \mathrm{Li} \mathrm{X}$ (2016) $\mathrm{H}_{2} \mathrm{O}_{2}$ regulates root system architecture by modulating the polar transport and redistribution of auxin. J Plant Biol 59:260-270. https://doi.org/10.1007/s12374-016-0052-1

Sun HW, Tao JY, Liu SJ, Huang SJ, Chen S, Xie XN, Yoneyama K, Zhang YL, Xu GH (2014) Strigolactones are involved in phosphateand nitrate-deficiency-induced root development and auxin transport in rice. J Exp Bot 65:6735-6746. https://doi.org/10.1093/jxb/eru029

Tsukagoshi H (2016) Control of root growth and development by reactive oxygen species. Curr Opin Plant Biol 29:57-63. https://doi. org/10.1016/j.pbi.2015.10.012

Vieten A, Sauer M, Brewer PB, Friml J (2007) Molecular and cellular aspects of auxin-transport-mediated development. Trends Plant Sci 12:160-168. https://doi.org/10.1016/j.tplants.2007.03.006

Wang LJ, Jiang WB, Zhang Z, Yao QH, Matsui H, Ohara H (2003) Biosynthesis and physiological activities of 5-aminolevulinic acid (ALA) and its potential application in agriculture. Plant Physiol Commun 39:185-192. https://doi.org/10.13592/j.cnki. ppj.2003.03.001

Wang LJ, Jiang WB, Liu H, Liu WQ, Kang L, Hou XL (2005) Promotion by 5 -aminolevulinic acid of germination of pakchoi (Brassica campestris ssp. chinensis var. communis Tsen et Lee) seeds under salt stress. J Integr Plant Biol 47:1084-1091. https://doi.org/10.111 1/j.1744-7909.2005.00150.x

Wang QN, An B, Wei YX, Reiter RJ, Shi HT, Luo HL, He CZ (2016) Melatonin regulates root meristem by repressing auxin synthesis and polar auxin transport in Arabidopsis. Front Plant Sci 7:1882. https:// doi.org/10.3389/fpls.2016.01882

Wei ZY, Li J (2016) Brassinosteroids regulate root growth, development, and symbiosis. Mol Plant 9:86-100. https://doi.org/10.1016/j. molp.2015.12.003

Wei ZY, Zhang ZP, Lee MR, Sun YP, Wang LJ (2012) Effect of 5-aminolevulinic acid on leaf senescence and nitrogen metabolism of pakchoi under different nitrate levels. J Plant Nutr 35:49-63. https ://doi.org/10.1080/01904167.2012.631666

Wu Y, Liao WB, Dawuda MM, Hu LL, Yu JH (2019) 5-Aminolevulinic acid (ALA) biosynthetic and metabolic pathways and its role in higher plants: a review. Plant Growth Regul 87:357-374. https:// doi.org/10.1007/s10725-018-0463-8

Xu WF, Jia LG, Shi WM, Liang JS, Zhou F, Li QF, Zhang JH (2013) Abscisic acid accumulation modulates auxin transport in the root tip to enhance proton secretion for maintaining root growth under moderate water stress. New Phytol 197:139-150. https://doi.org/10.1111/ nph.12004

Zhao YY, Yan F, Hu LP, Zhou XT, Zou ZR, Cui LR (2015) Effects of exogenous 5-aminolevulinic acid on photosynthesis, stomatal conductance, transpiration rate, and PIP gene expression of tomato seedlings subject to salinity stress. Genet Mol Res 14:6401-6412. https://doi.org/10.4238/2015.June.11.16

Zhen A, Bie ZL, Huang Y, Liu ZX, Fan ML (2012) Effects of 5-aminolevulinic acid on the $\mathrm{H}_{2} \mathrm{O}_{2}$-content and antioxidative enzyme gene expression in NaCl-treated cucumber seedlings. Biol Plant 56:566-570. https://doi.org/10.1007/s10535-012-0118-y

Zheng XH, Miller ND, Lewis DR, Christians MJ, Lee KH, Muday GK, Spalding EP, Vierstra RD (2011) AUXIN UP-REGULATED F-BOX PROTEIN1 regulates the cross talk between auxin transport and cytokinin signaling during plant root growth. Plant Physiol 156:1878-1893. https://doi.org/10.1104/pp.111.179812

Publisher's Note Springer Nature remains neutral with regard to jurisdictional claims in published maps and institutional affiliations. 